\title{
Uso de avaliação do desempenho para prescrição de dispositivos de tecnologia assistiva
}

\section{Performance evaluation for prescription of assistive technology devices}

\author{
Claudiene Aline Fonsêca de Souza ${ }^{1}$, Marcos Ferreira Calixto ${ }^{2}$, \\ Marianne Pinheiro Marques $^{3}$, Ana Cláudia Barroso de Sá Oliveira ${ }^{4}$, \\ Ana Cristina de Jesus Alves ${ }^{5}$
}

http://dx.doi.org/10.11606/issn.2238-6149.v29i1p34-40

Souza CAF, Calixto MF, Marques MP, Sá Oliveira ACB, Alves ACJ. Uso de avaliação do desempenho para prescrição de dispositivos de tecnologia assistiva. Rev Ter Ocup Univ São Paulo. 2018 jan.-abr.;29(1):34-40.

RESUMO: Introdução: A indicação e implementação da Tecnologia Assistiva (TA), quando realizada de forma integrada, pode auxiliar na prescrição de dispositivos que favoreçam a maior participação nas atividades cotidianas, atenuando o impacto imposto pelas limitações funcionais de indivíduos com deficiências físicas. Objetivo: verificar as contribuições do uso da Medida Canadense de Desempenho Ocupacional (COPM), fundamentada na prática centrada no cliente, no momento da indicação de TA. Procedimentos metodológicos: Trata-se de um estudo descritivo transversal realizado com 31 indivíduos com deficiência física de um hospital público do Distrito Federal. A coleta de dados ocorreu por meio da aplicação da avaliação COPM, sendo realizada a categorização das TA a partir do levantamento das áreas de desempenho mais comprometidas. Resultados: Do total de participantes, 51,6 \% $(\mathrm{n}=16)$ solicitaram alguma indicação e/ou confecção de TA. A área de autocuidado foi apontada como a mais comprometida $(96,7 \%)$, concentrando também, maior número de solicitações de TA. Conclusão: Este estudo pode contribuir com as pesquisas que investigam o uso de avaliações para a indicação de dispositivos e, espera-se que novas pesquisas possam dar continuidade a este processo, priorizando a participação ativa do indivíduo no momento de avaliar as necessidades do uso da TA.

DESCRITORES: Atividades cotidianas; Equipamentos de autoajuda; Pessoas com deficiência; Reabilitação; Avaliação de resultados (cuidados de saúde).
Souza CAF, Calixto MF, Marques MP, Sá Oliveira ACB, Alves ACJ. Performance evaluation for prescription of assistive technology devices. Rev Ter Ocup Univ São Paulo. 2018 Jan.-Apr.;29(1):34-40.

ABSTRACT: Introduction: The prescription and implementation of Assistive Technology (AT), when done correctly, can alleviate the impact functional limitations have on physically disabled individuals, providing them with greater participation in everyday activities. Objective: To verify the contributions of using the Canadian Occupational Performance Measure (COPM), which is focused on the client's performance, when prescribing the use of AT. Methods: This is a research of descriptive transversal character, carried out with 31 patients from a public hospital in the Federal District. Data collection was carried out through the application of the COPM evaluation, and the study of the most compromised performance areas was useful for the categorization of the Assistive Technology devices. Results: From the total of participants, $51.6 \%(\mathrm{n}=16)$ asked for indication and/or production of an AT device. The self-care area was the most compromised $(96.7 \%)$, and also had the most requests for AT devices. Conclusion: This study can contribute to studies that investigate the use of evaluations for the prescription of devices. We hope that new researches can give this process continuity, prioritizing an active participation of the client when evaluating the need for an AT device.

KEYWORDS: Activities of daily living; Self-help devices; Disabled persons; Rehabilitation; Outcome assessment (Health care).

Este artigo é parte do Trabalho de Conclusão de Curso da primeira autora apresentado à Universidade de Brasília - Faculdade de Ceilândia como requisito para obtenção do título de Bacharel em Terapia Ocupacional, professora orientadora Dra. Ana Cristina de Jesus Alves.

1. Terapeuta Ocupacional, Brasília, DF, BR. ORCID: https://orcid.org/0000-0002-1055-7633. E-mail: claudienealine@gmail.com

2. Terapeuta Ocupacional da Secretaria de Estado de Saúde do Distrito Federal, SES/DF, Brasília, DF, BR. ORCID: https://orcid.org/00000003-4876-0909. E-mail: marcos.calixto.to@gmail.com

3. Terapeuta Ocupacional, Mestranda no Programa de Ciências da Reabilitação da Faculdade de Ceilândia - UnB/FCE, Brasília, DF, BR. ORCID: https://orcid.org/0000-0001-9508-3096. E-mail: marianne_pc@hotmail.com

4. Terapeuta Ocupacional da Secretaria de Estado de Saúde do Distrito Federal, SES/DF, Brasília, DF, BR. Especialista em Reabilitação Cognitiva pelo NEPNEURO/Goiânia, GO. ORCID: https://orcid.org/0000-0003-1029-8296. E-mail: claubarroso.7@gmail.com

5. Docente do Programa de Pós-Graduação em Ciência da Reabilitação e do curso de Terapia Ocupacional da Universidade de Brasília Faculdade de Ceilândia. ORCID: https://orcid.org/0000-0003-4361-1365. E-mail: crisjalves@hotmail.com

Endereço para correspondência: Claudiene Aline Fonsêca de Souza. CCSW 05, Bl A, Apt. 244. Edifício Ômega Center, Sudoeste. Brasília, DF, BR. CEP: 70.680-550. 


\section{INTRODUÇÃO}

I ndivíduos que apresentam limitações motora, cognitiva e/ou sensorial podem, frequentemente, vivenciar dificuldades na execução das atividades de vida diária ou necessitar de ajuda de terceiros na realização das mesmas. Estas limitações acabam prejudicando seu desempenho ocupacional, contribuindo assim, para o declínio na qualidade de vida e exclusão social ${ }^{1}$.

Neste sentido, o novo modelo da Classificação Internacional de Funcionalidade, Incapacidade e Saúde (CIF) apresenta a deficiência e a incapacidade não apenas como uma condição de saúde/doença, mas também como uma situação ligada à participação e ao contexto ambiental e social do sujeito. Tal modelo pode direcionar a oferta ampliada de serviços de saúde, além da criação de diretrizes sensíveis à condição funcional do sujeito ${ }^{2}$.

Pensando-se, no contexto como um importante componente, a Tecnologia Assistiva (TA) é apontada na CIF como um fator ambiental que pode atuar como facilitador ao melhor desempenho do indivíduo com alguma deficiência. Com isso, o uso de dispositivos de TA busca atenuar o impacto imposto pelas limitações funcionais, proporcionando maior participação nas atividades cotidianas destes sujeitos ${ }^{1}$.

A CIF classifica a TA, chamada de tecnologia de assistência, como um componente ambiental podendo ser para uso pessoal na vida diária, para mobilidade e transporte, comunicação, educação, trabalho, atividades culturais e religiosas e projetos arquitetônicos ${ }^{2}$.

A abordagem, denominada de Prática Centrada no Cliente $^{3}$, coloca o sujeito como elemento essencial no processo de avaliação e intervenção, levando em consideração suas expectativas quanto à intervenção, e respeitando sua autonomia enquanto coparticipante no processo. O profissional de reabilitação, ao utilizar a prática centrada no cliente, deve considerar o que $o$ cliente deseja e precisa a curto, médio e longo prazo, incluindo-o como sujeito ativo no processo terapêutico ${ }^{4}$.

Seguindo esta abordagem, o modelo Matching Person and Technology - MPT sugere que o profissional que indica TA identifique o dispositivo mais adequado para determinada pessoa e alinhe as intervenções com as prioridades do sujeito 5 . Este modelo, específico para a área, descreve três aspectos a serem considerados no momento da indicação de um dispositivo, sendo eles: a) os fatores psicossociais; b) os fatores ambientais e; c) os fatores particulares dos dispositivos.

Segundo Tam et al. ${ }^{6}$, tendo como referência a prática centrada no cliente, os autores apresentaram a Medida Canadense de Desempenho Ocupacional (COPM) como instrumento potencial na indicação e implementação destes dispositivos de TA. A utilização do instrumento permite levantar aspectos quanto ao desempenho funcional e satisfação, comparar resultados em decorrência de uma intervenção, e neste caso, determinar alterações significativas na realização das atividades cotidianas após o uso dos dispositivos de $\mathrm{TA}^{6}$.

Entretanto, a produção científica nos países em desenvolvimento é escassa nesta área, gerando diversos questionamentos aos profissionais sobre formas de indicação e os reais benefícios advindos da indicação destas tecnologias? ${ }^{7}$. Além disso, a ausência de uma linha de base que oriente os profissionais no processo avaliativo dificulta estabelecer prioridades de indicação das TA conforme a real necessidade dos clientes ${ }^{7}$.

Esta lacuna no conhecimento, segundo Garcia e Galvão-Filho ${ }^{7}$, pode também contribuir negativamente na definição de políticas públicas, assim como na configuração adequada de iniciativas de apoio e promoção de projetos sobre o tema.

Assim, o objetivo deste estudo foi verificar as contribuições do uso da avaliação COPM, no momento da indicação de TA para pessoas com deficiência física. Trata-se de um instrumento traduzido e validado no Brasil, e em países desenvolvidos, que se fundamenta na prática centrada no cliente. Tem-se também como propósito, levantar as principais demandas funcionais dos indivíduos com deficiência física e a indicação das TA para esta população.

\section{PROCEDIMENTOS METODOLÓGICOS}

O presente trabalho, caracteriza-se como um estudo descritivo transversal, que compõe uma pesquisa maior intitulada "Indicação e implementação de tecnologia assistiva com indivíduos com deficiência fisica", a qual foi submetida ao Comitê de Ética e Pesquisa da Fundação de Ensino e Pesquisa (FEPECS), recebendo parecer favorável $\left(n^{\circ} 417.214 / 13\right)$.

Nesta pesquisa foram preconizados os termos da Resolução $n^{\circ}$ 466/12 do Conselho Nacional de Saúde, que determina as normas regulamentadoras de pesquisas envolvendo seres humanos. Os participantes que concordaram em participar assinaram o Termo de Consentimento Livre e Esclarecido (TCLE), sendo 
informados sobre os objetivos e procedimentos da pesquisa.

Foi utilizada uma amostragem de conveniência que diz respeito à utilização de um grupo amostral específico ${ }^{8}$. Participaram da pesquisa indivíduos jovens e adultos com lesão neurológica que estavam em acompanhamento no programa de reabilitação de um Hospital Público do Distrito Federal. Tal hospital é considerado referência na região Centro-Oeste no tratamento e reabilitação precoce de indivíduos pós Lesões Neurológicas centrais e periféricas, fazendo a cobertura de todo o Distrito Federal e Sistema Único de Saúde, sendo admitidos aproximadamente dez mil indivíduos ao ano nos programas de reabilitação desta instituição.

O período de coleta foi de agosto a novembro de 2014 e no mês de maio de 2015. Como critérios de inclusão, os participantes deveriam estar em atendimento no setor de reabilitação, acompanhados pela terapeuta ocupacional do hospital, apresentar deficiência física, ter a capacidade de comunicação verbal inteligível e autojulgamento preservados. Foram excluídos os indivíduos que apresentaram aspectos clínicos instáveis ou que estivessem com alta agendada nos próximos dias.

A coleta de dados foi realizada uma vez na semana, na sala de Terapia Ocupacional, sendo realizada primeiramente a caracterização da amostra por meio da aplicação de questionário que buscou identificar a idade, sexo e diagnóstico clínico.

Para a investigação de demandas ao uso de TA foi aplicada, com todos os participantes indicados, a avaliação de desempenho ocupacional, a Medida Canadense de Desempenho Ocupacional (COPM). A avaliação tem um caráter de instrumento individualizado, semiestruturado, em que o sujeito pontua as atividades que considera mais importante no seu cotidiano, destacando as dificuldades na realização destas ${ }^{9}$, além de abranger três áreas de desempenho ocupacional: atividades de autocuidado, produtividade e de lazer ${ }^{9,10}$.

Em relação ao direcionamento à indicação de TA, o pesquisador e o cliente identificavam, dentre as cinco principais atividades cotidianas, aquelas que se beneficiariam do uso de um dispositivo de TA para melhora do desempenho e satisfação em sua realização.

Após identificação do interesse pela aquisição da $\mathrm{TA}$, os clientes foram orientados à compra do dispositivo ou à confecção do mesmo junto ao grupo de apoio do projeto, composto por pesquisadores e estudantes de graduação em Terapia Ocupacional. O treino para o uso do dispositivo foi realizado ao longo do estudo sob a responsabilidade dos pesquisadores envolvidos.
As demandas ocupacionais, não relacionadas ao processo de indicação e/ou confecção de dispositivos, foram acompanhadas pela terapeuta ocupacional do serviço, utilizando outros recursos, dando continuidade ao processo de reabilitação.

Para a análise dos dados relacionados às áreas de desempenho ocupacional e a necessidade de TA foram utilizados os dados obtidos na avaliação COPM, e a categorização dos participantes e das TA foram utilizadas planilhas do Excel 2010 para posterior discussão.

\section{RESULTADOS}

Participaram 31 indivíduos, sendo 54,8\% $(\mathrm{n}=17)$ participantes do sexo masculino e 45,2 \% $(n=14)$ do sexo feminino, com média de idade 39 anos. Quanto ao diagnóstico registrado em prontuário, a maioria apresentou Traumatismo Crânio Encefálico 29,0\% (n=9) e Acidente Vascular Cerebral 29,0\% ( $\mathrm{n}=9)$, seguido por Lesão Medular 19,3\% ( $\mathrm{n}=6)$, síndrome de Guillian-Barré $9,6 \%(\mathrm{n}=3)$, Mielopatia $6,4 \%(\mathrm{n}=2)$ e Ataxia apendicular $6,4 \%(n=2)$.

Em relação aos dados obtidos na aplicação do instrumento de avaliação COPM, observou-se que dos 31 participantes, 93,5\% $(n=29)$ indicaram alguma limitação no desempenho ocupacional. Destes, 51,6\% $(n=16)$ solicitaram alguma indicação e/ou confecção de TA.

Todos os participantes $(n=31)$ relataram ter comprometimento em seu desempenho ocupacional. Destes, $96,7 \% \quad(n=30)$, referiram limitações nas atividades de autocuidado nas seguintes categorias em ordem de prioridade para o cliente: cuidados pessoais, $83,8 \%(\mathrm{n}=26)$, mobilidade funcional, $64,5 \%(\mathrm{n}=20)$ e comunicação oral e escrita $9,6 \%(n=3)$.

As limitações na área de lazer foram indicadas por $16,1 \%(n=5)$ dos participantes, os quais priorizaram as atividades de socialização e recreação. Percebe-se ainda que apenas $3,1 \%(\mathrm{n}=1)$ dos participantes destacou a área de produtividade, ressaltando as atividades do trabalho. Diante da identificação de limitações funcionais por todos os participantes, apenas $51,6 \% \quad(n=16)$ deles solicitaram algum tipo de T.A. No total, foram indicados 17 dispositivos de TA, no qual, um mesmo participante recebeu 2 dispositivos. Desta maneira, foram indicados 11 tipos diferentes de TA, concentrados principalmente na área de autocuidado, seguida pela produtividade e lazer.

O Quadro 1 apresenta os dispositivos de TA indicados e confeccionados para cada participante. 
Souza CAF, et al. Avaliação de desempenho e tecnologia assistiva. Rev Ter Ocup Univ São Paulo. 2018 jan./abr:;29(1):34-40.

Quadro 1 - Tecnologias Assistivas indicadas e confeccionadas de acordo com as demandas funcionais.

\begin{tabular}{|l|l|l|l|}
\hline $\begin{array}{l}\text { Área de desempenho } \\
\text { ocupacional }\end{array}$ & Tecnologia assistiva & Confecção e/ou indicação & Participante \\
\hline \multirow{5}{*}{ Cuidados Pessoais } & Substituidor de preensão para esponja de banho & Confeccionada & P1, P3, P16 \\
\cline { 2 - 4 } & Esponja para banho de cabo longo & Indicação para compra & P18 \\
\cline { 2 - 4 } & Engrossador de talher com substituidor de preensão & Confeccionada & P1, P3, P7 \\
\cline { 2 - 4 } & Colher com peso & Confeccionada & P8 \\
\cline { 2 - 4 } & Apoio para tronco para cadeira de rodas & Confeccionada & P1 \\
\cline { 2 - 4 } & Faixa para apoio dos pés para cadeira de rodas & Confeccionada & P1 \\
\cline { 2 - 4 } & Mesa de apoio para cadeira de rodas & Confeccionada & P20 \\
\cline { 2 - 4 } & Cunha de posicionamento no leito & Confeccionada & P19, P25 \\
\cline { 2 - 4 } & Órtese de posicionamento punho e mão & Indicação oficina de órtese e prótese & Ponfeccionada \\
\hline Produtividade & Pulseira com peso & Indicação & P30 \\
\hline Lazer & Comunicação Alternativa & & \\
\hline
\end{tabular}

Fonte: Elaboração própria.

O Quadro 1 mostra que, dentre os 11 tipos de dispositivos indicados, os mais solicitados foram os substituidores de preensão para banho e os engrossadores com substituidores de preensão para talher, seguido pela pulseira com peso e órtese de posicionamento.

Vale ressaltar que, do total dos dispositivos indicados, $88,2 \%(\mathrm{n}=15)$ das TA foram desenvolvidos pelos pesquisadores deste estudo e, apenas $11,8 \%(n=2)$ dos dispositivos foram encaminhados para compra ou aquisição.

\section{DISCUSSÃO}

Os dados mostraram que 48,4\% $\quad(n=15)$ dos participantes apresentaram lesões traumáticas como Traumatismo Crânio Encefálico 29,0\% (n=9) e Lesão Medular 19,4\% $(n=6)$. Os dados sobre a causa da deficiência podem estar relacionados ao alto índice de violência da região. De acordo com dados do Mapa de Violência de 2012 é possível esboçar a evolução da unidade federada do Distrito Federal - DF, destacando-se um crescimento acelerado da violência na região e um ritmo de crescimento que distancia progressivamente o DF das taxas nacionais de homicídios ${ }^{11}$.

Neste sentido, é importante a continuidade de implementação de políticas públicas que ofereçam ao indivíduo condições sociais e de saúde e que possibilitem e estimulem a participação das pessoas com alguma limitação².

Os resultados também mostraram que 100\% dos participantes com lesões neurológicas referiram apresentar limitações em uma ou mais áreas de desempenho ocupacional. Esse achado corrobora com a literatura ao indicar que as atividades de desempenho ocupacional são apontadas como essenciais, fazendo parte da vida de qualquer sujeito ${ }^{12}$. Porém, não se pode descartar que as atividades ocupacionais estão relacionadas às diversas funções do indivíduo e, portanto, torna-se importante considerar as habilidades físicas, a condição intelectual, o atributo de automanutenção, as atividades sociais, o conhecimento de si mesmo e o nível emocional do indivíduo ${ }^{2,13,14}$.

Neste sentido, o estudo comprova estes achados ao mostrar que 96,8\% $(n=30)$ dos participantes com lesões neurológicas referiram ter demandas ocupacionais na área de autocuidado, a qual corresponde às atividades de cuidados pessoais, mobilidade funcional e independência fora de casa. Dentre as áreas de desempenho ocupacionais mais citadas, às relacionadas aos cuidados pessoais representaram $80,6 \%$ $(\mathrm{n}=25)$ e a área de mobilidade funcional foi apontada por $61,3 \%(n=19)$ dos participantes.

Possivelmente, o cuidado com o próprio corpo assumiu destaque por ser considerado fundamental para um convívio social, além de possibilitar sobrevivência básica e o bem estar ${ }^{15,16}$. Quanto à demanda de mobilidade, os pesquisadores atribuem um menor número, possivelmente, ao fato dos participantes já apresentarem algum tipo de mobilidade, seja pela aquisição prévia a este estudo de cadeira de rodas ou, devido à restrição ao leito que os clientes estavam sujeitos.

Notou-se que as limitações em áreas relacionadas ao lazer e produtividade foram relatadas em menor frequência. Martinelli ${ }^{12}$ apontou em seu estudo, que as atividades de lazer estão diretamente relacionadas às possibilidades de autorrealização, escolhas e interesse individual ${ }^{17,18}$. Já a área de produtividade está relacionada à significação do 
trabalho para o indivíduo e, como esta pessoa pode ser instituída como sujeito, a garantir sua identidade e seu reconhecimento social ${ }^{19}$.

Assim, considerando-se que a Instituição pesquisada tem caráter de atendimento a indivíduos com sequelas neurológicas agudas e em contexto de internação, podese hipotetizar que as prioridades dos indivíduos estavam voltadas para o desempenho de atividades de autocuidado e que, a percepção e interesse relacionados à produtividade e de lazer estavam comprometidos ou tinham pouca relevância naquele momento de internação ${ }^{17,20}$.

Na população alvo pesquisada, apenas $51,6 \%(n=16)$ solicitaram a indicação e/ou confecção de TA. A literatura aponta que, a fase inicial de reabilitação, embora crucial, é marcada por condições de mudanças que trazem medos e/ ou angústias, dificuldade em expressar-se espontaneamente podendo fazer com que o indivíduo tome uma atitude passiva perante os cuidados recebidos ${ }^{17,18}$.

O contexto de inserção dos participantes desta pesquisa, neste caso, em processo de internação, pode dificultar o reconhecimento das dificuldades funcionais, as prioridades de aquisições ocupacionais e a indicação da necessidade de um auxílio, adaptação ou mesmo busca de soluções para sua nova condição. Autores ${ }^{2}$ reforçaram esta ideia, afirmando que, a aceitação ou não de TA estará diretamente relacionada ao nível funcional em que se encontra o indivíduo, seu autojulgamento quanto às limitações e expectativas de retorno a uma rotina que foi rompida pós-lesão ${ }^{16}$.

Dentre as solicitações de TA, 11 tipos de dispositivos foram indicados, sendo os mais comuns: adaptações para alimentação, para o banho e para a cadeira de rodas. Destaca-se que, embora existam políticas que determinam o financiamento e a aquisição de TA no Brasil, a maior parte dos dispositivos solicitados pelos participantes deste estudo não são contemplados pelas políticas, sendo preconizados apenas dispositivos de mobilidade (cadeiras, órtese, próteses, automóveis) e de comunicação (computadores, tablets), ou seja, os de alto custo e que já estavam em posse dos participantes desta pesquisa. Mesmo assim, os dados mostram que os dispositivos de mobilidade, presentes neste estudo, como a cadeira de rodas, necessitaram de ajustes e adaptações para o melhor posicionamento e mobilidade funcional.

Nota-se que $88,2 \%(\mathrm{n}=15)$ dos dispositivos foram confeccionados pelos pesquisadores. Este dado pode indicar a dificuldade que o indivíduo com deficiência tem em adquirir todas as TA que auxiliem o melhor desempenho em atividades básicas, como autocuidado, lazer e trabalho, que ainda não estão contempladas em lei, configurando como direito.
Em relação ao uso da COPM como uma avaliação para a indicação de TA, foi observado que ela pode nortear a indicação dos dispositivos, a partir da identificação das áreas de desempenho comprometidas e da satisfação do indivíduo em realizá-las.

Mesmo não sendo uma avaliação específica para indicar TA ela auxiliou os pesquisadores ao fornecer-lhes dados quanti-qualitativos. Estudos em países desenvolvidos vêm utilizando esta avaliação na prática centrada no cliente para indicação e mensuração do impacto dos dispositivos de TA no cotidiano da pessoa com deficiência, reforçando os resultados encontrados no presente estudo ${ }^{7,21}$.

De acordo com alguns autores ${ }^{6}$, poucas são as pesquisas que exibem sistematizações de indicação de dispositivos de TA nos países em desenvolvimento e/ou a investigação sobre real repercussão deste dispositivo na participação dos sujeitos. Isto se faz necessário, visto a quantidade de dispositivos que são indicados, seu custo e crescente aumento das politicas públicas que subsidiam a aquisição de TA no Brasil.

Outro fator importante, que deve ser destacado sobre o uso do instrumento de avaliação COPM, é o fato de o instrumento considerar a autopercepção do indivíduo em relação às suas atividades ocupacionais e sua satisfação, para que, a partir disso, ele possa ser protagonista de seu plano terapêutico, elencando junto ao terapeuta suas prioridades e recursos terapêuticos a serem utilizados ${ }^{22}$.

No entanto, vale destacar que, para os indivíduos que se apresentam em contexto social restrito, neste caso, hospitalizados e em situação de insegurança, as oportunidades para manifestar sua vontade, na maioria das vezes podem também estar limitadas. Os indivíduos, ao serem considerados incapazes ou com limitações, percebem seu poder de escolha restringido, configurando como barreira à sua liberdade em conduzir sua própria vida ${ }^{12}$.

Os modelos teóricos mais atuais que fazem referência à reabilitação e uso de TA têm preconizado a participação do indivíduo em todo o processo, seja na avaliação, na definição de objetivos, escolha dos recursos, no treinamento e medidas de resultados ${ }^{5,21}$. Assim, o processo de avaliação das necessidades e indicação de TA no contexto hospitalar devem também não apenas considerar as características perceptíveis das limitações funcionais, mas também as características pessoais e contextuais, incluindo as extra-hospitalares.

Outro ponto a ser destacado no uso da COPM, é que o instrumento permite avaliar alterações na autopercepção do indivíduo em relação ao seu desempenho num espaço de tempo. Neste sentido, configura-se como limitação deste estudo, a não utilização desta avaliação para verificar 
mudanças no desempenho, a partir do uso de uma TA que poderia favorecer o desempenho funcional do indivíduo.

No entanto, estudos ${ }^{23-25}$ que buscaram por modelos teóricos e avaliações para indicação de TA mostraram que, dentre os referenciais que não são específicos de TA, o modelo conceitual de saúde descrito pela CIF e a avaliação COPM, estão entre os três instrumentos mais utilizados mundialmente como norteadores para indicação de TA. Esses dados, assim como os achados deste estudo, reforçam que o uso de referenciais que focam fatores ambientais e de participação, como os trazidos pela CIF e, a prática centrada no cliente, como contemplada pela COPM, têm se tornado relevante no processo de avaliação, reabilitação e inclusão da pessoa com deficiência. Os achados deste estudo puderam mostrar, assim como a CIF, que os conceitos de funcionalidade e saúde devem extrapolar a condição de doença e/ou deficiência, e abranger também a participação e o contexto ambiental e social do sujeito, já que foi comprovada a grande demanda para estas áreas.

Atualmente, já existem avaliações específicas para indicação de TA no Brasil, tais como: a Avaliação de Tecnologia Assistiva - Predisposição ao Uso, a ATD $\mathrm{PA} \mathrm{Br}^{21}$ e a Quebec User Evaluation of Satisfaction with Assistive Technology, a QUEST 2.0, as quais não estavam disponíveis no período deste estudo. Tais avaliações têm como base teórica a relação multidirecional de componentes de saúde estabelecida pela CIF, sendo que a primeira avaliação segue o modelo teórico específico de TA, o MPT, o qual também foi fundamentado no modelo de funcionalidade proposto pela Organização Mundial da Saúde ${ }^{5,21,23}$. Assim, sugere-se que, a partir deste trabalho, novos estudos sejam realizados a fim dar continuidade às investigações sobre o uso de avaliação, prescrição, confecção, treino, eficácia e satisfação no uso destes dispositivos para indivíduos com alguma deficiência, podendo-se agora pensar em associar avaliações de TA de desempenho e satisfação já disponíveis no Brasil.

\section{CONCLUSÃO}

Considera-se que este estudo alcançou os objetivos propostos baseado nos fundamentos da prática centrada no cliente e no desempenho ocupacional. Identificou-se que as variáveis investigadas pelo instrumento puderam fornecer subsídios para a indicação de TA.

Este estudo pode identificar contribuições e barreiras em relação ao uso da COPM na prescrição da TA, com a população de jovens e adultos com deficiência física em um contexto hospitalar.

Pode-se também apresentara importância dada pelos participantes, às limitações nas atividades de desempenho ocupacional, principalmente as de autocuidado, principal área em que as TA foram indicadas.

Sugere-se que o tema aqui apresentado não se esgote e que mais pesquisas possam ser conduzidas para investigar o uso da COPM em um processo longitudinal na indicação da TA, em contextos diferentes ao hospitalar e com o uso conjunto com avaliações especificas de TA.

Autoria e Indicação de Responsabilidade: Claudiene Aline Fonsêca de Souza, Marcos Ferreira Calixto, Marianne Pinheiro Marques, Ana Cláudia Barroso de Sá Oliveira e Ana Cristina de Jesus Alves - participaram da concepção e delineamento da pesquisa. Claudiene Aline Fonsêca de Souza, Marcos Ferreira Calixto, Marianne Pinheiro Marques - participaram da coleta e da análise dos dados. Todos os pesquisadores participaram da redação e revisão do artigo. Claudiene Aline Fonsêca de Souza e Ana Cristina de Jesus Alves - participam da aprovação da versão a ser publicada.

\section{REFERÊNCIAS}

1. Braccialli LMP. Tecnologia Assistiva: Perspectiva de qualidade de vida para pessoas com deficiência. In: Vilarta R, Gutierrez GL, Carvalho TH, Gonçalves A, editor. Qualidade de vida e novas tecnologias. Campinas; 2007. p.105-13.

2. Castaneda L, Bergmann A, Bahia L. A Classificação Internacional de Funcionalidade, Incapacidade e Saúde: uma revisão sistemática de estudos observacionais. Rev Bras Epedemiol. 2014;17(2):437-51. doi: 10.1590/18094503201400020012.
3. Sumsion T. Prática baseada no cliente na terapia ocupacional - guia para implementação. São Paulo: Roca; 2003.

4. Carleto DGS, Souza AC, Silva M, Cruz DMC, Andrade VS. Estrutura da prática da terapia ocupacional: domínio e processo. Rev Triang. 2010;3(2):57-147 [citado 10 abr. 2015]. Disponível em: http://www.uftm.edu.br/ revistaeletronica/index.php/revistatriangulo/article/ view/150/177. 
5. Scherer MJ. Assessing the benefits of using assistive technologies and other supports for thinking, remembering and learning. Disabil Rehabil. 2005;27(13):731-9. doi: 10.1080/09638280400014816.

6. Tam C, Archer J, Mays J, Skidmore G. Measuring the outcomes of word cueing technology. Can J Occup Ther. 2005;72(5):3018. doi: 10.1177/000841740507200507.

7. Garcia JC, Galvão Filho TA. Pesquisa Nacional de Tecnologia Assistiva. Instituto de Tecnologia Social. São Paulo: ITS BRASIL/MCTI-SECIS, 2012 [citado 10 abr. 2015]. Disponível em: http://www.santoandre.sp.gov.br/pesquisa/ebooks/368505. PDF.

8. Creswell J. Projeto de pesquisa: métodos qualitativo, quantitativo e misto. 3a ed. Porto Alegre: Artmed; 2010.

9. Pollock N, McColl MA, Carswell A. Medida de Performance Ocupacional Canadense. In: Sunsion T, editor. Prática baseada no cliente na terapia ocupacional: guia para implementação. São Paulo: Roca; 2003. p.183-204.

10. Magalhães LC, Magalhães LV, Cardoso AA, organizadores. Medida Canadense de Desempenho Ocupacional (COPM) Versão brasileira. Belo Horizonte: UFMG; 2009.

11. Waiselfisz JJ. Mapa da violência 2012. Os novos padrões da violência homicida no Brasil. São Paulo: Instituto Sangari; 2012. p.115-6 [citado 11 jun. 2016]. Disponível em: http:// www.mapadaviolencia.org.br/pdf2012/mapa2012_df.pdf.

12. Martinelli SA. A importância de atividades de lazer na terapia ocupacional. Cad Ter Ocup UFSCar (São Carlos). 2011;19(1):111-8. Disponível em: http://www. cadernosdeterapiaocupacional.ufscar.br/index.php/cadernos/ article/view/429/31.

13. Pereira DC, Ruzzi Pereira A, Pereira PE, Trevisan ER. Desempenho ocupacional de adolescentes de um Centro de Atenção Psicossocial Infanto-Juvenil (CAPSI). Rev Ter Ocup São Paulo. 2014;25(1):11-7. doi: http://dx.doi.org/10.11606/ issn.2238-6149.v25i1p11-17.

14. Perlini NMOG, Mancussi e Faro AC. Cuidar de pessoa incapacitada por acidente vascular cerebral no domicílio: o fazer do cuidador familiar. Rev Esc Enferm USP 2005;39(2):154-63. doi: http://dx.doi.org/10.1590/S008062342005000200005 .

15. Machado WCA, Scramin AP. Cuidado multidimensional para e com pessoas tetraplégicas: re-pensando o cuidar em enfermagem. Ciên Cuidado Saúde. 2005;4(2):189-97. doi: http://dx.doi.org/10.4025/cienccuidsaude.v4i2.5248.
16. Mello MAF, Mancini MC. Métodos e técnicas de avaliação nas áreas de desempenho ocupacional. In: Cavalcanti A, Galvão C, editor. Terapia ocupacional: fundamentos e prática. São Paulo: Guanabara; 2007. p.49-54.

17. Machado WCA, Scramin AP. (In)dependência funcional na dependente relação de homens tetraplégicos com seus (in)substituíveis pais/cuidadores. Rev Esc Enferm USP. 2010;44(1):53-60. doi: http://dx.doi.org/10.1590/S008062342010000100008 .

18. Diogo MJD. A dinâmica dependência-autonomia em idosos submetidos à amputação de membros inferiores. Rev Latinoam Enfermagem (Ribeirão Preto). 1997;5(1):59-64. doi: http:// dx.doi.org/10.1590/S0104-11691997000100007.

19. Oliveira BRG; Murofuse NT. Acidentes de trabalho e doença ocupacional: estudo sobre o conhecimento do trabalhador hospitalar dos riscos à saúde de seu trabalho. Rev. Latino-Am. Enfermagem (Ribeirão Preto). 2001;9(1):109-15. doi: http:// dx.doi.org/10.1590/S0104-11692001000100016.

20. Delsim JC. Análises de mesas auxiliares (de refeição) para indivíduos utilizadas no ambiente hospitalar e proposta de requisitos de projeto para um novo modelo [Dissertação]. São Carlos: Universidade de São Paulo; 2011.

21. Alves ACJ. Avaliação de tecnologia assistiva predisposição ao uso: ATD PA Br: versão brasileira. Brasília: Editora Universidade de Brasília; 2017 [citado 13 jun. 2018]. Disponível em: http://repositorio.unb.br/bitstream/10482/31065/1/ RELATORIO_AvaliacaodetecnologiaassistivaATDPA.pdf.

22. Eyssen ICJM, Steultjens MPM, Oud TAM, Bolt M, Maasdan A, Dekker J. Responsiveness of the Canadian Occupational Performance Measure. J Rehabil Respons Dev. 2011;48(5):51728. doi: 10.1682/JRRD.2010.06.0110.

23. Scherer MJ; Sax CL. Measures of assistive technology predisposition and use. In: Mpofu E; Oakland T. editors. Rehabilitation and health assessment: applying ICF guidelines. EUA: Springer Publishing Company; 2010. p.229-54.

24. Alves ACJ, Matsukura TS. Theoretic models for indication and implementation of assistive technology. Cad Ter Ocup UFSCar (São Carlos). 2016;24(3):591-9. doi: http://dx.doi. org/10.4322/0104-4931.ctoAO1078.

25. Alves ACJ, Matsukura TS. Review about assessments for indication of assistive technology. Rev Ter Ocup Univ São Paulo. 2014;25(2):199-207. doi: http://dx.doi.org/10.11606/ issn.2238-6149.v25i2p199-207. 